\title{
Cost Analysis of Enhanced Recovery Programs in Colorectal, Pancreatic, and Hepatic Surgery: A Systematic Review
}

\author{
Gaëtan-Romain Joliat $^{1}$ (1) $\cdot$ Martin Hübner $^{1} \cdot$ Didier Roulin $^{1} \cdot$ Nicolas Demartines $^{1}$
}

Published online: 29 October 2019

(C) Société Internationale de Chirurgie 2019

\begin{abstract}
Background Enhanced recovery programs (ERPs) have been shown to improve postoperative outcomes after abdominal surgery. This study aimed to review the current literature to assess if ERPs in colorectal, pancreas, and liver surgery induce cost savings.

Methods A systematic review was performed including prospective and retrospective studies comparing conventional management versus ERP in terms of costs. All kinds of ERP were considered (fast-track, ERAS ${ }^{\circledR}$, or homemade protocols). Studies with no mention of a clear protocol and no reporting of protocol compliance were excluded. Results Thirty-seven articles out of 144 identified records were scrutinized as full articles. Final analysis included 16 studies. In colorectal surgery, two studies were prospective (1 randomized controlled trial, RCT) and six retrospective, totaling 1277 non-ERP patients and 2078 ERP patients. Three of the eight studies showed no difference in cost savings between the two groups. The meta-analysis found a mean cost reduction of USD3010 (95\% CI: $5370-650, p=0.01$ ) in favor of ERP. Among the five included studies in pancreas surgery (all retrospective, 552 non-ERP vs. 348 ERP patients), the mean cost reduction in favor of the ERP group was USD7020 (95\% CI: $11,600-2430, p=0.003$ ). In liver surgery, only three studies (two retrospective and 1 RCT, 180 non-ERP vs. 197 ERP patients) were found, which precluded a sound cost analysis.

Conclusions The present systematic review suggests that ERPs in colorectal and pancreas surgery are associated with cost savings compared to conventional perioperative management. Cost data in liver surgery are scarce.
\end{abstract}

\section{Introduction}

Enhanced recovery programs (ERPs) are multimodal management protocols for surgical patients [1]. ERPs are developed using evidence-based best practices [2]. The

This article was presented in parts at the Annual Congress of the Swiss Surgical Society in Basel, Switzerland, May 16-18, 2018.

Nicolas Demartines

demartines@chuv.ch

1 Department of Visceral Surgery, Lausanne University Hospital CHUV, Rue du Bugnon 46, 1011 Lausanne, Switzerland main aim of these programs is to improve patient recovery by applying a standardized and comprehensive perioperative management [3].

ERPs have been shown to induce important benefits in major abdominal surgeries [4]. Several studies showed a decrease in length of hospital stay (LoS) and/or a reduction in postoperative complications [5-9]. This standardization of care has been shown to improve clinical outcomes in abdominal surgery as well as in other types of surgery [10].

In addition, several articles lately suggested that ERP could induce cost savings in various types of surgery [11-13]. 
The aim of the present study was to review the current literature to assess the impact on costs of ERP in colorectal, pancreas, and liver surgery.

\section{Materials and methods}

A systematic review of the current literature was performed. The reporting of this manuscript followed the PRISMA and MOOSE guidelines for systematic review and meta-analysis $[14,15]$.

\section{Inclusion and exclusion criteria}

Studies comparing the costs of ERP to conventional management in colorectal, pancreas, and liver surgery were eligible. Inclusion criteria were a clear description of the enhanced recovery protocol and mention of the compliance to the pathway. All types of studies were considered (retrospective or prospective). Studies without control group were excluded. All types of enhanced recovery protocols $\left(\right.$ ERAS $^{\circledR}$, fast-track, or home made) and all surgery types were considered as long as the inclusion criteria were fulfilled. Only articles in English were included [16].

\section{Search methods}

Articles were searched using MEDLINE/PubMed, Ovid, Isis, the Cochrane Library, Google Scholar, Web of Knowledge, and Embase. Articles published between January 1980 and June 2018 were included. Cross-referencing of all eligible articles was also performed to improve the search. MeSH terms used were "colon," "pancreas," and "liver," and free text terms used were "enhanced recovery" and "costs."

\section{Primary and secondary outcomes}

Primary outcome was the overall costs per patient. Secondary outcomes were the costs of specific areas during the hospital stay (e.g., laboratory, radiology, medical care, or housing) and the costs of readmission.

\section{Data collection}

Two reviewers (GRJ and DR) independently performed the search of eligible studies. Results were compared between the two authors. In the absence of consensus, the senior author (ND) made the final decision about inclusion or not. Characteristics of the studies were extracted from every manuscript. If data were not clear or incomplete, corresponding authors were contacted to gain further information. Costs were expressed as mean and standard deviation.
For comparison and meta-analysis, all costs were converted into US dollars (USD). The exchange rate of each currency to USD was calculated as on June 30, 2018 ( 1 Euro $=1.23$ USD, 1 Can Dollar $=0.76$ USD, and $1 \mathrm{GBP}=1.31 \mathrm{USD})$.

\section{Risk of bias assessment}

The quality of the included studies was evaluated using the Newcastle-Ottawa scale and Downs and Black scale $[17,18]$.

\section{Statistical analysis}

Heterogeneity was evaluated in terms of clinical and statistical heterogeneity, as well as methodological diversity. A Chi-squared test was performed to assess the statistical heterogeneity. Heterogeneity quantification (inconsistency across the studies) was measured by the $I^{2}$ value, defining the percentage of variability due to heterogeneity rather than chance.

When feasible, meta-analysis of costs (continuous variables) was undertaken using inverse variance method following the Cochrane Collaboration recommendations. If statistical heterogeneity (i.e., $I^{2}>50 \%$ ) was substantial between the studies, a random-effect model was used to calculate the forest plots instead of a fixed-effect model. When necessary medians with range or interquartile range were transformed to means and standard deviation according to published formulas [19]. A $P$ value $\leq 0.05$ was considered statistically significant (corresponding to a $\mathrm{Z}$ overall effect $\geq 1.96$ ). Review Manager 5.3@ for Mac OS X (The Nordic Cochrane Centre for The Cochrane Collaboration, 2014) was used for the forest plots.

\section{Results}

The process of the systematic review is shown in Fig. 1 (flowchart according to the PRISMA guidelines). After initial search, 144 studies were retrieved. After duplicate removal, 37 studies were scrutinized for inclusion [11-13, 20-53].

\section{Included and excluded studies}

Sixteen studies were finally included: eight studies in colorectal surgery $[11,20-26]$, five in pancreatic surgery $[12,38-41]$, and three in liver surgery $[13,49,50]$. Three studies were prospective, while 13 studies were retrospective. Table 1 summarizes the characteristics of the included studies. In total, colorectal studies included 1277 non-ERP patients versus 2078 ERP patients, pancreas 
Fig. 1 PRISMA flowchart of the study

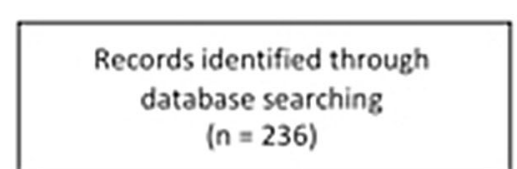

( $n=236$ )

\section{Additional records identified}

through other sources

( $n=10$ )

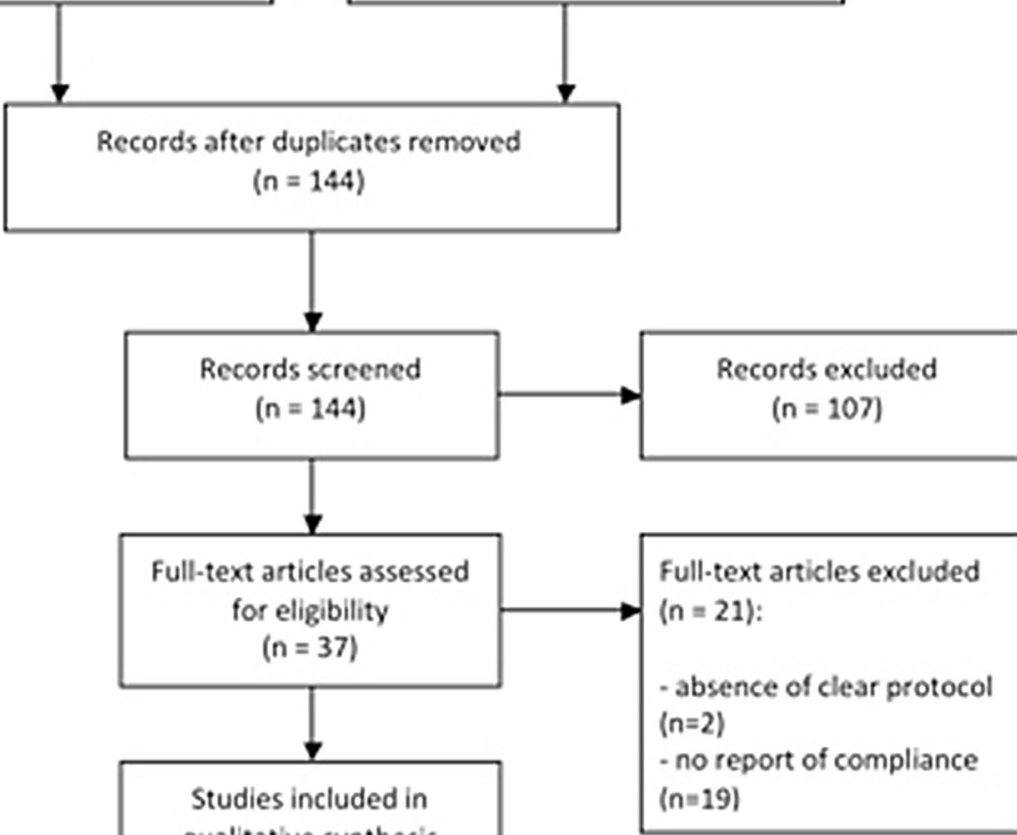

qualitative synthesis

$(n=16)$

Table 1 Characteristics of the included studies

\begin{tabular}{|c|c|c|c|c|c|c|}
\hline First author & Year & Study origin & Study design & $\begin{array}{l}\text { Patient number ERP } \\
\text { versus non-ERP }\end{array}$ & $\begin{array}{l}\text { Quality scores } \\
\text { (Newcastle-Ottawa, } \\
\max .4 / 2 / 3 \text { ) }\end{array}$ & $\begin{array}{l}\text { Downs and black } \\
\text { (max. 32) }\end{array}$ \\
\hline \multicolumn{7}{|l|}{ Colorectal } \\
\hline Vlug [21] & 2011 & Netherlands & $\mathrm{RCT}$ & 207 versus 193 & $4 / 2 / 3$ & 23 \\
\hline Roulin [20] & 2013 & Switzerland & Before and after & 50 versus 50 & $4 / 2 / 3$ & 19 \\
\hline Geltzeiler [26] & 2014 & USA & Before and after & 176 versus 68 & $4 / 2 / 3$ & 18 \\
\hline Miller [22] & 2014 & USA & Before and after & 142 versus 99 & $4 / 2 / 3$ & 19 \\
\hline Thiele [23] & 2015 & USA & Before and after & 109 versus 98 & $4 / 2 / 3$ & 17 \\
\hline Wick [24] & 2015 & USA & Before and after & 330 versus 310 & $4 / 2 / 2$ & 19 \\
\hline Lee [11] & 2015 & Canada & Prospective cohort & 95 versus 95 & $4 / 2 / 3$ & 21 \\
\hline Nelson [25] & 2016 & Canada & Before and after & 983 versus 350 & $4 / 2 / 3$ & 19 \\
\hline \multicolumn{7}{|l|}{ Pancreas } \\
\hline Joliat [38] & 2015 & Switzerland & Before and after & 74 versus 87 & $4 / 2 / 3$ & 19 \\
\hline Williamsson [12] & 2015 & Sweden & Before and after & 50 versus 50 & $4 / 2 / 3$ & 19 \\
\hline Richardson [40] & 2015 & UK & Before and after & 22 versus 44 & $4 / 2 / 2$ & 16 \\
\hline Morgan [39] & 2016 & USA & Before and after & 81 versus 297 & $4 / 2 / 3$ & 19 \\
\hline Kagedan [41] & 2017 & Canada & Before and after & 121 versus 74 & $4 / 2 / 3$ & 20 \\
\hline \multicolumn{7}{|l|}{ Liver } \\
\hline $\mathrm{He}$ [13] & 2015 & China & $\mathrm{RCT}$ & 48 versus 38 & $4 / 2 / 3$ & 16 \\
\hline Page [50] & 2015 & USA & Before and after & 42 versus 75 & $4 / 2 / 3$ & 17 \\
\hline Joliat [49] & 2016 & Switzerland & Before and after & 74 versus 100 & $4 / 2 / 3$ & 19 \\
\hline
\end{tabular}

$R C T$ randomized controlled trial 
studies 552 non-ERP versus 348 ERP patients, and liver studies 180 non-ERP versus 197 ERP patients.

Twenty-one studies did not fulfill the inclusion criteria and were therefore excluded (Fig. 1). Among these 21 studies, 19 studies did not disclose the compliance rate [27-35, 37, 42, 43, 45-48, 51-53], and two did not have a clear protocol [36, 44]. Of note, two additional studies $[54,55]$ were not considered for inclusion as they originated from the same cohort as the article by Nelson et al. [25].

\section{Compliance}

Five studies (two in colorectal, one in pancreas, and two in liver surgery) mentioned the mean overall compliance to the ERP (range from 70 to $74 \%$ ) [20, 21, 38, 49, 50]. The study by Nelson et al. on colorectal surgery found a median compliance to the ERP of $60 \%$ [25]. Seven articles showed the compliance to specific elements of the pathway [11-13, 22, 23, 26, 40]. Kagedan et al. scored the compliance based on four predefined goals (tolerance of clear fluids on POD1, discharge from monitored care setting by POD2, ambulation in room on POD1, and tolerance of solid diet by POD4, 50\% of the patients fulfilled the four goals) [41]. In one study, the compliance was described in the post-article discussion [39], and in another study, compliance was described in the methods to have been measured but was not clearly reported [24].

\section{Missing data}

Data on overall costs were missing in one study [50]. In the study by Geltzeiler et al., only the mean difference between non-ERP and ERP patients was mentioned with no details on the means of overall costs for each group [26]. It was not possible to retrieve these data from the authors. In the study by Nelson et al., the used methodology (cost difference calculated by multiplying the difference of LoS between non-ERP and ERP patients by the estimated unit costs of hospital stay) did not permit to have mean overall costs for non-ERP and ERP patients [25].

\section{Quality assessment}

Quality scores of the studies are also included in Table 1. Median Newcastle-Ottawa and Downs and Black scores were $4 / 2 / 3$ and 19 , respectively. Overall quality of the included studies was judged good.

\section{Overall and specific costs}

Table 2 shows the individual results of overall costs for colorectal, pancreas, and liver surgery. In colorectal surgery, three studies found significant mean differences of costs in favor of ERP [11, 23, 24], two studies found a mean difference in favor of ERP but no $p$-value was mentioned $[25,26]$, and three studies did not find statistically significant cost differences between ERP and nonERP patients [20-22]. Miller et al. showed a gain in the ERP group for specific costs (room-related and pharmacy costs) [22]. For pancreas surgery, 4 out of 5 studies found a significant economic advantage of ERP [12, 39-41]. In a study from Switzerland, overall hospital costs were not statistically different between the two groups, but costs of anesthesia/operation room, medication, and laboratory were lower in ERP patients [38]. In liver surgery, one study found a significant mean difference in favor of ERP for overall costs [13], while one study found significant cost savings for medical supply, pharmacy, laboratory, and therapy costs in favor of ERP [50]. The study where overall costs were similar found savings for ERP patients in radiology costs [49].

The forest plot for colorectal studies showed a mean difference of USD3010 in favor of ERP (95\% CI: $5370-650, p=0.01)$. For pancreas surgery, the meta-analysis showed a mean difference of USD7020 (95\% CI: $11,600-2430, p=0.003$ ) in favor of ERP. Forest plots are shown in Figs. 2 and 3. For liver surgery, meta-analysis was not performed as only two studies expressed mean and standard deviation of overall costs. It was not possible to obtain the overall cost data from the authors of the other study [50].

\section{Readmission costs}

Roulin et al. and Vlug et al. for colorectal surgery included the costs of readmission within 30 postoperative days in the overall hospital costs without specifying the costs of the readmission stay $[20,21]$. Richardson et al. specified the overall costs in pancreatic surgery with and without readmission costs [40]. Including the readmission costs, median overall costs were in the study by Richardson et al. USD11,463 in the non-ERP group versus USD8797 in the ERP group ( $p=0.0004)$ [40]. Similarly, Kagedan et al. also reported the overall costs in pancreatic surgery with and without readmission costs and found a cost decrease even when readmission costs were included (USD22,703 vs. USD12,637, $p=0.016$ ) [41].

\section{Discussion}

In this systematic review and meta-analysis, ERP induced mean cost savings per patient of USD3010 and USD7020 in colorectal and pancreas surgery, respectively. Cost data in liver surgery were insufficient to draw conclusions. 
Table 2 Total overall cost results of the included studies

\begin{tabular}{|c|c|c|c|}
\hline & Mean costs ERP and non-ERP in USD & Mean difference in USD & $P$ value \\
\hline \multicolumn{4}{|l|}{ Colorectal } \\
\hline Vlug $^{\text {cf }}[21]$ & $13,031 / 14,719^{\mathrm{a}}$ & $-1688^{\mathrm{b}}$ & 0.56 \\
\hline Roulin $^{\mathrm{cf}}[20]$ & $31,142 / 33,173$ & -2031 & 0.621 \\
\hline Geltzeiler [26] & N/A & -4803 & N/A \\
\hline Miller [22] & $18,377 / 20,537$ & -2161 & 0.31 \\
\hline Thiele [23] & $18,777 / 25,334$ & -6567 & $<0.001$ \\
\hline Wick [24] & $9036 / 10,933$ & -1897 & 0.013 \\
\hline Lee $^{\mathrm{cf}}[11]$ & $11,074 / 13,343$ & -2269 & $<0.05$ \\
\hline Nelson [25] & N/A & -2806 to -5898 & N/A \\
\hline \multicolumn{4}{|l|}{ Pancreas } \\
\hline Joliat $^{\mathrm{c}}[38]$ & $68,982 / 78,500$ & -9518 & 0.273 \\
\hline Williamsson $^{\mathrm{c}}[12]$ & $12,792 / 17,928^{\mathrm{a}}$ & $-5136^{\mathrm{b}}$ & $<0.001$ \\
\hline Richardson $^{c}[40]$ & $8797 / 11,305$ & -2508 & 0.0003 \\
\hline Morgan [39] & $23,308 / 27,388$ & -4080 & $<0.0001$ \\
\hline Kagedan $^{c}[41]$ & $11,915 / 19,557$ & -7642 & 0.024 \\
\hline \multicolumn{4}{|l|}{ Liver } \\
\hline $\mathrm{He}^{\mathrm{d}}[13]$ & $7742 / 9470$ & -1728 & 0.03 \\
\hline Page $^{\mathrm{e}}[50]$ & N/A & N/A & N/A \\
\hline Joliat $^{\mathrm{c}}$ [49] & $47,633 / 52,098$ & -4465 & 0.467 \\
\hline
\end{tabular}

N/A not available. In the study by Vlug et al., only laparoscopy patients were taken into account

${ }^{\mathrm{a}}$ Median

${ }^{b}$ Difference of medians

${ }^{\mathrm{c}}$ Costs converted in USD

${ }^{\mathrm{d}}$ Currency not specified in the manuscript and no response from the authors

${ }^{\mathrm{e}}$ Only partial costs were mentioned

${ }^{\mathrm{f}}$ Costs of readmissions are included in the overall costs

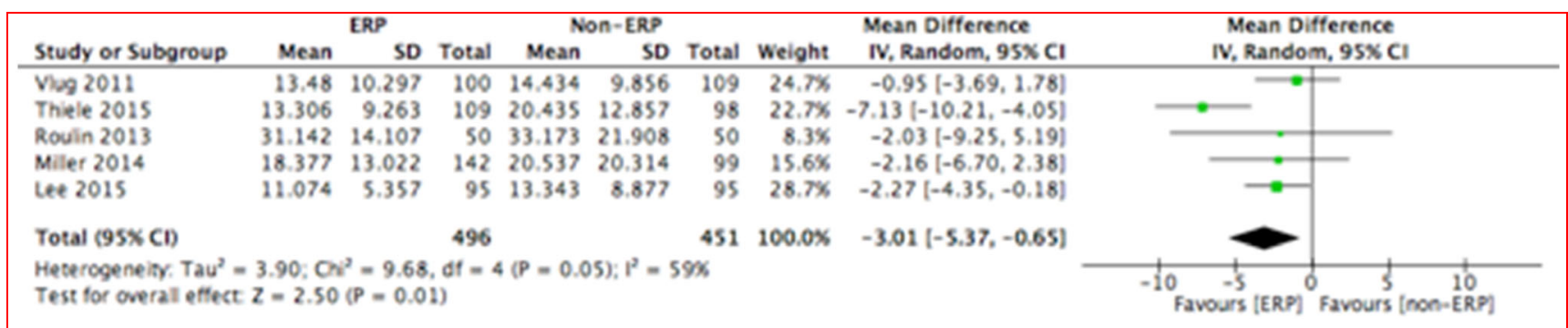

Fig. 2 Forest plot of mean overall costs in colorectal surgery (in 1000 USD)

\begin{tabular}{|c|c|c|c|c|c|c|c|c|c|c|}
\hline \multirow[b]{2}{*}{ Study or Subgroup } & \multicolumn{3}{|c|}{ ERP } & \multicolumn{3}{|c|}{ Non-ERP } & \multirow[b]{2}{*}{ Weight } & \multirow{2}{*}{$\begin{array}{l}\text { Mean Difference } \\
\text { IV, Random, } 95 \% \mathrm{CI}\end{array}$} & \multirow{2}{*}{\multicolumn{2}{|c|}{$\begin{array}{l}\text { Mean Difference } \\
\text { IV, Random, } 95 \times C I\end{array}$}} \\
\hline & Mean & SD & Total & Mean & SD & Total & & & & \\
\hline Joliat 2015 & 68.982 & 4.266 & 74 & 78.5 & 6.986 & 87 & $28.9 \%$ & $-9.52[-11.28,-7.76]$ & $\because$ & \\
\hline Willamson 2015 & 11.25 & 11.519 & 50 & 20.285 & 12.749 & so & $22.8 \%$ & $-9.04[-13.80,-4.27]$ & & \\
\hline Kagedan 2017 & 11.915 & 10.029 & 121 & 19.557 & 26.05 & 74 & $19.4 x$ & $-7.64[-13.84,-1.44]$ & & \\
\hline Richardson 2015 & 8.797 & 2.312 & 22 & 11.305 & 4.88 & 44 & $28.9 x$ & $-2.51[-4.24,-0.77]$ & $\rightarrow-$ & \\
\hline Total $(95 \% \mathrm{Cl})$ & & & 267 & & & 255 & $100.0 x$ & $-7.02[-11.60,-2.43]$ & & \\
\hline \multicolumn{9}{|c|}{$\begin{array}{l}\text { Heterogeneity: Tau }=18.11 ; \mathrm{Ch}^{2}=32.59, \mathrm{df}=3(\mathrm{P}<0.00001) ; \mathrm{r}^{2}=91 \mathrm{x} \\
\text { Test for overall effect: } Z=3.00(\mathrm{P}=0.003)\end{array}$} & $-10 \quad-5$ & Favours [non-ERP] \\
\hline
\end{tabular}

Fig. 3 Forest plot of mean overall costs in pancreas surgery (in 1000 USD) 
In colorectal surgery, the economic gain can be explained by several points. In the study by Thiele et al., cost reduction was explained by a decrease in complication rate [23]. Moreover, the authors highlighted the fact that reduction of LoS permitted to have more admissions, therefore increasing the revenue of an institution operating near capacity. Several studies showed that a diminution of LoS usually correlates with cost diminution, but only with limited impact $[56,57]$. It is not the shorter LoS per se that induces cost gains but the supplementary gains of additional hospitalization allowed by shorter LoS, also called costs of opportunity [23]. Moreover, complication reduction-as reported in two studies - allows saving money and resources $[23,25]$. The study by Roulin et al. found specific gains in the medication, laboratory, and radiology costs [20]. The standardization of care through ERAS ${ }^{\circledR}$ implementation avoided unnecessary and expensive exams without increasing the complication rate. In a systematic review by Lee et al. published in Annals of Surgery in 2014, 10 studies were included and found cost savings for ERP but evidence was judged low and limited [58]. Of note, most of the included studies of Lee's meta-analysis did not report compliance rate to the ERP pathways. Compliance was chosen as an inclusion criterion in the present study because it measures how a specific pathway is followed. Furthermore, compliance has been linked to complications, LoS and also reflects the practical implementation and enforcement of a pathway [59, 60]. The results of this article confirm and reinforce the previous findings by Lee et al. Similar results were found in 2015 by Lemanu et al. in their systematic review with a range of savings from 153 to 6537 euros [61].

Almost all included pancreas studies showed significant cost savings after ERP implementation, with a mean difference of USD7020 per patient in favor of ERP. Economic gains following ERP implementation was explained in the article by Williamsson et al. by the decrease in LoS and performed radiological exams induced by standardized management [12]. Richardson et al. argued that cost reduction was due to LoS decrease as well as bed day savings [40]. Kagedan et al. found particular cost decrease in laboratory tests, investigation, pharmacy, imaging, and patient food, which explains the decrease in total hospital costs in favor of ERP [41]. Having a clear and standardized protocol permits to avoid costly investigations such as clinically unnecessary laboratory tests or radiological imagings. The decrease in costs found in the present study correlates with the findings of the meta-analysis on ERP in non-colorectal surgery by Visioni et al. [62]. They found as secondary outcome that costs were decreased by a mean of USD5109 (inclusion of 10 randomized controlled trials of major abdominal surgery). Kagedan et al. performed a systematic review on the evidence of ERP in pancreas resection in 2015 and found only four studies assessing costs [63]. Two of them showed a cost benefit of ERP, while the two others did not find any difference in terms of costs. Already in 2013, Coolsen et al. found in their systematic review and meta-analysis on ERP in pancreas surgery 3 out of 4 studies showing cost reduction after ERP implementation [64]. Finally, Xiong et al. published in 2016 a systematic review on ERP in pancreatic surgery and found four studies mentioning in-hospital costs [65]. All of them showed a significant decrease in costs after ERP implementation.

ERP has only been recently applied to liver surgery with publication of the ERAS ${ }^{\circledR}$ guidelines in 2016 [66]. Data on costs of ERP implementation in liver surgery are presently very limited, as shown by the three included studies. These weak existing data showed a tendency toward economic benefit for ERP but it was not possible to draw a sound conclusion on the financial impact of ERP in liver surgery. Future studies are needed to confirm or disprove the current preliminary data. Several systematic reviews were performed [67-72]. Most of them did not include costs in the analysis [67-69, 71, 72]. Ahmed et al. performed a systematic review on fast-track in liver surgery, where only two articles discussed costs [70]. The first was the study by He et al. [13] included in this article, and the second found that fast-track management decreased hospital costs by $30 \%$ [52].

Stowers et al. found in their systematic review that ERPs were cost effective, but highlighted the fact that out-hospital cost data were missing and should be examined more in depth in the future [73]. In the present review, only one study assessed out-hospital costs for colorectal surgery [11]. Lee et al. interestingly found that ERP implementation decreased costs of productivity loss by a mean difference of 1062 Canadian dollars (95\% CI: 2054-74) and caregiver costs by a mean difference of 247 Canadian dollars (95\% CI: 463-49).

It is interesting to mention that among the six studies in colorectal and pancreas surgery that assessed specific cost categories $[11,20,22,24,38,41]$, four did not find a category where ERP was significantly more expensive [11, 22, 24, 41]. In the two remaining studies, Roulin et al. only found higher intraoperative costs for ERP due to laparoscopy increase and Joliat et al. found an increase in housing and administration costs for ERP due to temporal inflation and unrelated to ERP [20, 38]. It is always possible that a random effect might have played a role in these studies but this is unlikely considering that the majority of studies found better overall costs for ERP and the occurrences where ERP was found to be more expensive for specific area were infrequent. 
The overall quality of the evidence was judged good, but 13 studies were retrospective which can induce some bias in the analysis. Missing data were a limitation of the present study. Most included studies presented costs as secondary outcome (10/16 studies) $[12,13,21-23,25,26,39,40,50]$ and might have been underpowered to find a difference in costs. In addition, several studies did not perform a sample size calculation. Moreover, heterogeneity among studies was substantial, especially for the methodology of cost assessment (real costs vs. cost estimation) as reflected by the differences of procedure costs shown in Table 2. This highlights the need for standardization of cost reporting to improve future inter-study comparisons. However, the group of included articles was to the author opinion sufficiently homogeneous in terms of etiologies, included patients, ERP types, measured cost outcomes, and overall quality to perform a metaanalysis. Moreover, adding data of specific operations (e.g., colectomy, low anterior resection, distal pancreatectomy, or pancreatoduodenectomy) contributed to answer the wider question of ERP costs in colorectal, pancreas, and liver surgery (including all previously cited operations), which was the aim of the present study. Of course, one must be aware of this heterogeneity when interpreting the results. It is also important to mention that international cost comparison across different health care systems is really challenging because cost implications are largely dependent on the health care system. As a large number of heterogeneous variables (calculation methods, types of included costs, time period differences, or country specificities) were present among included studies, it was statistically not feasible to take all of them into account in the analysis. Included studies focused on various types of interventions, which renders the overall analysis more difficult. Nevertheless, the goal of this study was not to assess ERP costs for specific operations but rather to obtain a more global picture of ERP costs for surgical subspecialties such as colorectal and pancreas surgery. It was also not possible to assess the role that secular trend played in the included before and after ERP implementation studies. Finally, cost data on liver surgery were unfortunately sparse.

In conclusion and despite the above-mentioned limitations, ERP was associated with cost reduction in colorectal and pancreas surgery compared to non-ERP. Moreover, very few economic data are currently reported for ERP in liver surgery. For this reason, future studies are awaited to assess potential economic benefit of ERP in liver surgery, as well as in other specialties.

Acknowledgements We thank the authors of the included studies who provided additional data.
Funding None

Compliance with ethical standards

Conflict of interest None

\section{References}

1. Adamina M, Gié O, Demartines N et al (2013) Contemporary perioperative care strategies. Br J Surg 100:38-54

2. Wind J, Polle SW, Fung Kon Jin PHP et al (2006) Systematic review of enhanced recovery programmes in colonic surgery. $\mathrm{Br} \mathrm{J}$ Surg 93:800-809

3. Gatt M, Anderson ADG, Reddy BS et al (2005) Randomized clinical trial of multimodal optimization of surgical care in patients undergoing major colonic resection. $\mathrm{Br} \mathrm{J}$ Surg 92:1354-1362

4. Adamina M, Kehlet H, Tomlinson GA et al (2011) Enhanced recovery pathways optimize health outcomes and resource utilization: a meta-analysis of randomized controlled trials in colorectal surgery. Surgery 149:830-840

5. Grant MC, Yang D, Wu CL et al (2017) Impact of Enhanced recovery after surgery and fast track surgery pathways on healthcare-associated infections: results from a systematic review and meta-analysis. Ann Surg 265:68-79

6. Varadhan KK, Neal KR, Dejong CHC et al (2010) The enhanced recovery after surgery (ERAS) pathway for patients undergoing major elective open colorectal surgery: a meta-analysis of randomized controlled trials. Clin Nutr 29:434-440

7. Muller S, Zalunardo MP, Hubner M et al (2009) A fast-track program reduces complications and length of hospital stay after open colonic surgery. Gastroenterology 136:842-847

8. Gouvas N, Tan E, Windsor A et al (2009) Fast-track vs standard care in colorectal surgery: a meta-analysis update. Int $\mathrm{J}$ Colorectal Dis 24:1119-1131

9. Zhuang C-L, Ye X-Z, Zhang X-D et al (2013) Enhanced recovery after surgery programs versus traditional care for colorectal surgery: a meta-analysis of randomized controlled trials. Dis Colon Rectum 56:667-678

10. Nicholson A, Lowe MC, Parker J et al (2014) Systematic review and meta-analysis of enhanced recovery programmes in surgical patients. Br J Surg 101:172-188

11. Lee L, Mata J, Ghitulescu GA et al (2015) Cost-effectiveness of enhanced recovery versus conventional perioperative management for colorectal surgery. Ann Surg 262:1026-1033

12. Williamsson C, Karlsson N, Sturesson C et al (2015) Impact of a fast-track surgery programme for pancreaticoduodenectomy. Br J Surg 102:1133-1141

13. He F, Lin X, Xie F et al (2015) The effect of enhanced recovery program for patients undergoing partial laparoscopic hepatectomy of liver cancer. Clin Transl Oncol 17:694-701

14. Moher D, Liberati A, Tetzlaff J et al (2009) Preferred reporting items for systematic reviews and meta-analyses: the PRISMA statement. BMJ 339:b2535

15. Stroup DF, Berlin JA, Morton SC et al (2000) Meta-analysis of observational studies in epidemiology: a proposal for reporting. meta-analysis of observational studies in epidemiology (MOOSE) group. JAMA 283:2008-2012

16. Moher D, Pham B, Klassen TP et al (2000) What contributions do languages other than English make on the results of meta-analyses? J Clin Epidemiol 53:964-972

17. Ottawa Hospital Research Institute. http://www.ohri.ca/programs/ clinical_epidemiology/oxford.asp. Accessed 30 Jul 2018 
18. Downs SH, Black N (1998) The feasibility of creating a checklist for the assessment of the methodological quality both of randomised and non-randomised studies of health care interventions. J Epidemiol Community Health 52:377-384

19. Hozo SP, Djulbegovic B, Hozo I (2005) Estimating the mean and variance from the median, range, and the size of a sample. BMC Med Res Methodol 5:13

20. Roulin D, Donadini A, Gander S et al (2013) Cost-effectiveness of the implementation of an enhanced recovery protocol for colorectal surgery. Br J Surg 100:1108-1114

21. Vlug MS, Wind J, Hollmann MW et al (2011) Laparoscopy in combination with fast track multimodal management is the best perioperative strategy in patients undergoing colonic surgery: a randomized clinical trial (LAFA-study). Ann Surg 254:868-875

22. Miller TE, Thacker JK, White WD et al (2014) Reduced length of hospital stay in colorectal surgery after implementation of an enhanced recovery protocol. Anesth Analg 118:1052-1061

23. Thiele RH, Rea KM, Turrentine FE et al (2015) Standardization of care: impact of an enhanced recovery protocol on length of stay, complications, and direct costs after colorectal surgery. J Am Coll Surg 220:430-443

24. Wick EC, Galante DJ, Hobson DB et al (2015) Organizational culture changes result in improvement in patient-centered outcomes: implementation of an integrated recovery pathway for surgical patients. J Am Coll Surg 221:669-677

25. Nelson G, Kiyang LN, Crumley ET et al (2016) Implementation of enhanced recovery after surgery (ERAS) across a provincial healthcare system: the ERAS alberta colorectal surgery experience. World J Surg 40:1092-1103

26. Geltzeiler CB, Rotramel A, Wilson C et al (2014) Prospective study of colorectal enhanced recovery after surgery in a community hospital. JAMA Surg 149:955-961

27. King PM, Blazeby JM, Ewings P et al (2006) The influence of an enhanced recovery programme on clinical outcomes, costs and quality of life after surgery for colorectal cancer. Colorectal Dis 8:506-513

28. Stephen AE, Berger DL (2003) Shortened length of stay and hospital cost reduction with implementation of an accelerated clinical care pathway after elective colon resection. Surgery 133:277-282

29. Kariv Y, Delaney CP, Senagore AJ et al (2007) Clinical outcomes and cost analysis of a "fast track" postoperative care pathway for ileal pouch-anal anastomosis: a case control study. Dis Colon Rectum 50:137-146

30. Archibald LH, Ott MJ, Gale CM et al (2011) Enhanced recovery after colon surgery in a community hospital system. Dis Colon Rectum 54:840-845

31. Ren L, Zhu D, Wei Y et al (2012) Enhanced recovery after surgery (ERAS) program attenuates stress and accelerates recovery in patients after radical resection for colorectal cancer: a prospective randomized controlled trial. World J Surg 36:407-414

32. Sammour T, Zargar-Shoshtari K, Bhat A et al (2010) A programme of enhanced recovery after surgery (ERAS) is a costeffective intervention in elective colonic surgery. $\mathrm{N} \mathrm{Z} \mathrm{Med} \mathrm{J}$ 123:61-70

33. Pritts TA, Nussbaum MS, Flesch LV et al (1999) Implementation of a clinical pathway decreases length of stay and cost for bowel resection. Ann Surg 230:728-733

34. Lovely JK, Maxson PM, Jacob AK et al (2012) Case-matched series of enhanced versus standard recovery pathway in minimally invasive colorectal surgery. Br J Surg 99:120-126

35. Bosio RM, Smith BM, Aybar PS et al (2007) Implementation of laparoscopic colectomy with fast-track care in an academic medical center: benefits of a fully ascended learning curve and specialty expertise. Am J Surg 193:413-415
36. Archer SB, Burnett RJ, Flesch LV et al (1997) Implementation of a clinical pathway decreases length of stay and hospital charges for patients undergoing total colectomy and ileal pouch/anal anastomosis. Surgery 122:699-703

37. Tan JJY, Foo AYZ, Cheong DMO (2005) Colorectal clinical pathways: a method of improving clinical outcome? Asian J Surg 28:252-256

38. Joliat G-R, Labgaa I, Petermann D et al (2015) Cost-benefit analysis of an enhanced recovery protocol for pancreaticoduodenectomy. Br J Surg 102:1676-1683

39. Morgan KA, Lancaster WP, Walters ML et al (2016) Enhanced recovery after surgery protocols are valuable in pancreas surgery patients. J Am Coll Surg 222:658-664

40. Richardson J, Di Fabio F, Clarke H et al (2015) Implementation of enhanced recovery programme for laparoscopic distal pancreatectomy: feasibility, safety and cost analysis. Pancreatology 15:185-190

41. Kagedan DJ, Devitt KS, Tremblay St-Germain A et al (2017) The economics of recovery after pancreatic surgery: detailed cost minimization analysis of an enhanced recovery program. HPB (Oxford) 19:1026-1033

42. Shao Z, Jin G, Ji W et al (2015) The role of fast-track surgery in pancreaticoduodenectomy: a retrospective cohort study of 635 consecutive resections. Int J Surg 15:129-133

43. Dai J, Jiang Y, Fu D (2017) Reducing postoperative complications and improving clinical outcome: enhanced recovery after surgery in pancreaticoduodenectomy - a retrospective cohort study. Int J Surg 39:176-181

44. Porter GA, Pisters PW, Mansyur C et al (2000) Cost and utilization impact of a clinical pathway for patients undergoing pancreaticoduodenectomy. Ann Surg Oncol 7:484-489

45. Kennedy EP, Rosato EL, Sauter PK et al (2007) Initiation of a critical pathway for pancreaticoduodenectomy at an academic institution-the first step in multidisciplinary team building. J Am Coll Surg 204:917-923 (discussion 923-924)

46. Kennedy EP, Grenda TR, Sauter PK et al (2009) Implementation of a critical pathway for distal pancreatectomy at an academic institution. J Gastrointest Surg 13:938-944

47. Vanounou T, Pratt W, Fischer JE et al (2007) Deviation-based cost modeling: a novel model to evaluate the clinical and economic impact of clinical pathways. J Am Coll Surg 204:570-579

48. Kowalsky SJ, Zenati MS, Steve J et al (2018) A combination of robotic approach and eras pathway optimizes outcomes and cost for pancreatoduodenectomy. Ann Surg 269(6):1138-1145

49. Joliat G-R, Labgaa I, Hübner M et al (2016) Cost-benefit analysis of the implementation of an enhanced recovery program in liver surgery. World J Surg 40:2441-2450

50. Page AJ, Gani F, Crowley KT et al (2016) Patient outcomes and provider perceptions following implementation of a standardized perioperative care pathway for open liver resection. Br J Surg 103:564-571

51. Liang X, Ying H, Wang $\mathrm{H}$ et al (2016) Enhanced recovery program versus traditional care in laparoscopic hepatectomy. Medicine (Baltimore) 95:e2835

52. Sánchez-Pérez B, Aranda-Narváez JM, Suárez-Muñoz MA et al (2012) Fast-track program in laparoscopic liver surgery: theory or fact? World J Gastrointest Surg 4:246-250

53. Lin D-X, Li X, Ye Q-W et al (2011) Implementation of a fasttrack clinical pathway decreases postoperative length of stay and hospital charges for liver resection. Cell Biochem Biophys 61:413-419

54. Thanh NX, Chuck AW, Wasylak T et al (2016) An economic evaluation of the enhanced recovery after surgery (ERAS) multisite implementation program for colorectal surgery in Alberta. Can J Surg 59:415-421 
55. Nelson G, Kiyang LN, Chuck A et al (2016) Cost impact analysis of enhanced recovery after surgery program implementation in alberta colon cancer patients. Curr Oncol 23:e221-e227

56. Fine MJ, Pratt HM, Obrosky DS et al (2000) Relation between length of hospital stay and costs of care for patients with community-acquired pneumonia. Am J Med 109:378-385

57. Taheri PA, Butz DA, Greenfield LJ (2000) Length of stay has minimal impact on the cost of hospital admission. J Am Coll Surg 191:123-130

58. Lee L, Li C, Landry $\mathrm{T}$ et al (2014) A systematic review of economic evaluations of enhanced recovery pathways for colorectal surgery. Ann Surg 259:670-676

59. Berian JR, Ban KA, Liu JB et al (2017) Adherence to enhanced recovery protocols in NSQIP and association with colectomy outcomes. Ann Surg 269:486-493

60. Bakker N, Cakir H, Doodeman HJ et al (2015) Eight years of experience with enhanced recovery after surgery in patients with colon cancer: impact of measures to improve adherence. Surgery 157:1130-1136

61. Lemanu DP, Singh PP, Stowers MDJ et al (2014) A systematic review to assess cost effectiveness of enhanced recovery after surgery programmes in colorectal surgery. Colorectal Dis $16: 338-346$

62. Visioni A, Shah R, Gabriel E et al (2018) Enhanced Recovery after surgery for noncolorectal surgery?: a systematic review and meta-analysis of major abdominal surgery. Ann Surg 267:57-65

63. Kagedan DJ, Ahmed M, Devitt KS et al (2015) Enhanced recovery after pancreatic surgery: a systematic review of the evidence. HPB (Oxford) 17:11-16

64. Coolsen MME, van Dam RM, van der Wilt AA et al (2013) Systematic review and meta-analysis of enhanced recovery after pancreatic surgery with particular emphasis on pancreaticoduodenectomies. World J Surg 37:1909-1918
65. Xiong J, Szatmary P, Huang W et al (2016) Enhanced recovery after surgery program in patients undergoing pancreaticoduodenectomy: a PRISMA-Compliant systematic review and metaanalysis. Medicine (Baltimore) 95:e3497

66. Melloul E, Hübner M, Scott M et al (2016) Guidelines for perioperative care for liver surgery: enhanced recovery after surgery (ERAS) society recommendations. World J Surg 40:2425-2440

67. Hughes MJ, McNally S, Wigmore SJ (2014) Enhanced recovery following liver surgery: a systematic review and meta-analysis. HPB (Oxford) 16:699-706

68. Lei Q, Wang X, Tan S et al (2014) Fast-track programs versus traditional care in hepatectomy: a meta-analysis of randomized controlled trials. Dig Surg 31:392-399

69. Song W, Wang K, Zhang R-J et al (2016) The enhanced recovery after surgery (ERAS) program in liver surgery: a meta-analysis of randomized controlled trials. Springerplus 5:207

70. Ahmed EA, Montalti R, Nicolini D et al (2016) Fast track program in liver resection: a PRISMA-compliant systematic review and meta-analysis. Med (Baltimore) 95:e4154

71. Damania R, Cocieru A (2017) Impact of enhanced recovery after surgery protocols on postoperative morbidity and mortality in patients undergoing routine hepatectomy: review of the current evidence. Ann Transl Med 5:341

72. Page AJ, Ejaz A, Spolverato G et al (2015) Enhanced recovery after surgery protocols for open hepatectomy-physiology, immunomodulation, and implementation. J Gastrointest Surg 19:387-399

73. Stowers MDJ, Lemanu DP, Hill AG (2014) Health economics in Enhanced Recovery After Surgery programs. Can J Anaesth $62: 219-230$

Publisher's Note Springer Nature remains neutral with regard to jurisdictional claims in published maps and institutional affiliations. 